\title{
Linear and nonlinear optical responses in Kitaev spin liquids
}

\author{
Minoru Kanega, ${ }^{1, *}$ Tatsuhiko N. Ikeda $\odot,{ }^{2, \dagger}$ and Masahiro Sato ${ }^{1, \hbar}$ \\ ${ }^{1}$ Department of Physics, Ibaraki University, Mito, Ibaraki 310-8512, Japan \\ ${ }^{2}$ Institute for Solid State Physics, University of Tokyo, Kashiwa, Chiba 277-8581, Japan
}

(Received 15 January 2021; accepted 8 July 2021; published 28 July 2021)

\begin{abstract}
We theoretically study THz-light-driven high-harmonic generation (HHG) in the spin-liquid states of the Kitaev honeycomb model with a magnetostriction coupling between spin and electric polarization. To compute the HHG spectra, we numerically solve the Lindblad equation, taking account of the dissipation effect. We find that isotropic Kitaev models possess a dynamical symmetry, which is broken by a static electric field, analogous to HHG in electron systems. We show that the HHG spectra exhibit characteristic continua of Majorana fermion excitations, and their broad peaks can be controlled by applying static electric or magnetic fields. In particular, the magnetic-field dependence of the HHG spectra drastically differs from those of usual ordered magnets. These results indicate that an intense $\mathrm{THz}$ laser provides a powerful tool to observe dynamic features of quantum spin liquids.
\end{abstract}

DOI: 10.1103/PhysRevResearch.3.L032024

Introduction. Quantum spin liquids (QSLs) have attracted tremendous attention for decades as exotic states of matter. Many theoreticians have tried to find essential properties of QSLs, and it has been theoretically revealed that the low-energy excitations of QSLs are given by fractionalized particles, and the wave functions possess a topological nature [1-4].

Meanwhile, it has been recognized as notoriously difficult to identify QSLs experimentally because most of their thermodynamic quantities are featureless. Hence, the experiments have been done for their dynamical quantities. For instance, longitudinal [5-7] and transverse [8,9] transport phenomena have provided important information about lowenergy excitations in QSLs. For the Kitaev QSL [10-14], ferromagnetic $\alpha-\mathrm{RuCl}_{3}$ has been shown to exhibit several characteristic behaviors in, e.g., the thermal Hall effect [15], longitudinal thermal conductivity [7], and Raman scattering [16,17], and antiferromagnetic $\mathrm{YbCl}_{3}$ [18] has been expected to host QSL from neutron diffraction measurements. While these experimental results have been reasonably taken as evidence for QSLs, they are indirect, and active studies are ongoing to search for new ways to obtain further evidence.

One such direction is the nonlinear optical response at the $\mathrm{THz}$ frequency regime, which has been opened up by

\footnotetext{
*20nm010t@vc.ibaraki.ac.jp

†tikeda@issp.u-tokyo.ac.jp

${ }^{\ddagger}$ masahiro.sato.phys@vc.ibaraki.ac.jp
}

Published by the American Physical Society under the terms of the Creative Commons Attribution 4.0 International license. Further distribution of this work must maintain attribution to the author(s) and the published article's title, journal citation, and DOI. rapid development of $\mathrm{THz}$ laser technology [19-22]. Being at the energy scale of magnetic excitations, $\mathrm{THz}$ pulses are suitable for directly investigating and controlling quantum spin systems [23-46]. To detect crisp signatures of QSLs, the so-called THz two-dimensional coherent spectroscopy has been proposed [47] and theoretically analyzed in the Kitaev model [48]. However, in the Kitaev model, this method is based on third-order, rather than secondorder, optical response and thus it requires much stronger $\mathrm{THz}$ pulses for successful detection. The required intensity of $\mathrm{THz}$ pulses can be the bottleneck in experiments since spin-light couplings are generally much weaker than chargelight ones [37-43]. In fact, while high-harmonic generation (HHG) $[49,50]$ has been observed at $\mathrm{THz}$ frequencies in, e.g., Dirac electrons [51-53], only the second-order response has been reported [28] in magnetic insulators at present. Thus, another method based on lower-order nonlinear responses, if exists, should be useful for experimental verification of QSLs.

In this Letter, we show that a combination of an intense $\mathrm{THz}$ laser pulse and static electromagnetic fields uncovers characteristics of the Kitaev QSL through harmonic generation, including the second-order harmonic. We numerically analyze the HHG spectra of the Kitaev model with magnetostriction-type magnetoelectric (ME) coupling [54] with the quantum master equation approach $[41,46,55,56]$ to take account of dissipation effects. In addition to broad and continuous response functions characteristic of Majorana fermions, we find that static electric fields break some symmetry and activate the second-harmonic generation (SHG), and a static magnetic field causes an anomalous shift for the harmonic spectra. These findings indicate that nonlinear response to intense $\mathrm{THz}$ light gives us a powerful instrument for detecting dynamical features of Kitaev QSLs. Through this study, we will build a bridge between photoscience and QSLs. 


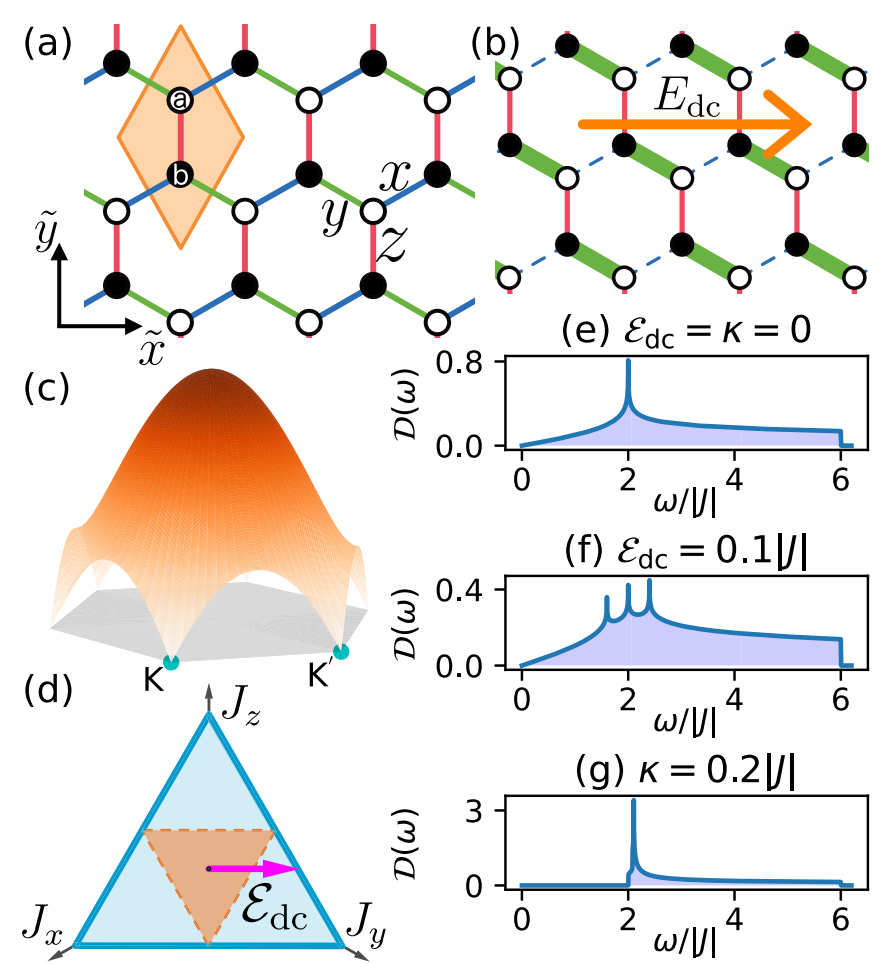

FIG. 1. (a) Lattice structure of the Kitaev model. Blue, green, and red lines, respectively, correspond to $x, y$, and $z$ bonds. (b) Kitaev model with a dimerization on $x$ and $y$ bonds, which is caused by a static electric field $E_{\mathrm{dc}}$ along the $\tilde{x}$ direction. (c) Gapless itinerant fermion band of the Kitaev model at $J_{x, y, z}=J$ and $E_{\mathrm{dc}}=\kappa=0$. (d) Ground-state phase diagram of the Kitaev model in $\left(J_{x}, J_{y}, J_{z}\right)$ space at $\kappa=0$. Orange and blue areas are, respectively, gapless and gapped QSLs. Application of $E_{\mathrm{dc}}$ induces $\left(J_{x}, J_{y}\right) \rightarrow\left(J_{x}-\mathcal{E}_{\mathrm{dc}}, J_{y}+\right.$ $\left.\mathcal{E}_{\mathrm{dc}}\right)$. (e)-(g) Density of states of itinerant fermions at $\left(\mathcal{E}_{\mathrm{dc}}, \kappa\right)=$ $(0,0),(0.1,0)$, and $(0,0.2)$ in $J<0$. For details of the density of states, see Figs. S8 and S9 of the supplemental material [57].

Kitaev Model and Methods. The Hamiltonian of the Kitaev model (see Fig. 1) [58] for this work is given by

$$
\hat{H}_{0}=-\sum_{\alpha,\left\langle\boldsymbol{r}, \boldsymbol{r}^{\prime}\right\rangle_{\alpha}} J_{\alpha} \sigma_{\boldsymbol{r}}^{\alpha} \sigma_{\boldsymbol{r}^{\prime}}^{\alpha}-\kappa \sum_{\mathrm{NNN}} \sigma_{\boldsymbol{r}}^{x} \sigma_{\boldsymbol{r}^{\prime}}^{y} \sigma_{\boldsymbol{r}^{\prime \prime}}^{z}-E_{\mathrm{dc}} \hat{P}
$$

with

$$
\hat{P}=\eta_{\mathrm{ms}}\left(-\sum_{\left\langle\boldsymbol{r}, \boldsymbol{r}^{\prime}\right\rangle_{x}} \sigma_{\boldsymbol{r}}^{x} \sigma_{\boldsymbol{r}^{\prime}}^{x}+\sum_{\left\langle\boldsymbol{r}, \boldsymbol{r}^{\prime}\right\rangle_{y}} \sigma_{\boldsymbol{r}}^{y} \sigma_{\boldsymbol{r}^{\prime}}^{y}\right),
$$

where $\sigma_{r}^{\alpha}(\alpha=x, y, z)$ is the Pauli matrix on the site $\boldsymbol{r}=$ $(j, k)$, and the spin-1/2 operator on $\boldsymbol{r}$ is $S_{r}^{\alpha}=\frac{\hbar}{2} \sigma_{r}^{\alpha}$ (we set $\hbar=1$ throughout the paper). $J_{\alpha}$ is the Ising coupling constant between nearest-neighboring spins on $\alpha$ bond $\left\langle\boldsymbol{r}, \boldsymbol{r}^{\prime}\right\rangle_{\alpha}$, and we mainly focus on the symmetric point $J=J_{x, y, z}$. The neighboring three-spin term [58] originates from the thirdorder perturbation with respect to a static Zeeman term $\mathcal{H}_{B}=$ $-g \mu_{B} \boldsymbol{B} \cdot \sum_{\boldsymbol{r}} \boldsymbol{\sigma}_{\boldsymbol{r}}^{\alpha}$ of an applied magnetic field $\boldsymbol{B}$ ( $g$ is the $g$ factor and $\mu_{B}$ is the Bohr magneton). The coupling constant is computed as $\kappa \sim\left(g \mu_{B}\right)^{3} B_{x} B_{y} B_{z} / J^{2}$ : For $|J| / k_{B}=10 \mathrm{~K}, \kappa \sim$ $0.1|J|$ corresponds to $|\boldsymbol{B}| \sim 1 T$. The $\kappa$ term is the leading term changing the Majorana-fermion dispersion (as one will see later) in the Zeeman interaction. The final term of Eq. (1) represents the coupling between electric polarization $\hat{P}$ and an applied static electric field $E_{\mathrm{dc}}$ along the $\tilde{x}$ direction [Figs. 1(b) and $1(\mathrm{~d})$ ]. Equation (2) assumes that $\hat{P}$ is proportional to dimerization along the $\tilde{x}$ direction. This kind of ME term appears in a class of multiferroic magnets and its typical origin is the spin-phonon coupling [54]. This dimerization makes the ground-state energy reduce (see the supplemental material [57]), and therefore this ME term may appear in a sort of real Kitaev-like material. In a class of multiferroics, the ME-coupling energy reaches that of the Zeeman term $[23-25,32,34,54,59,60]$, and thereby we have assumed that the effective coupling $\mathcal{E}_{\mathrm{dc}}=E_{\mathrm{dc}} \eta_{\mathrm{ms}}$ is the same order as $g_{0} \mu_{B} E_{\mathrm{dc}} / c$, with $c$ being the speed of light and $g_{0}=2$ : For $|J| / k_{B}=10 \mathrm{~K}, \mathcal{E}_{\mathrm{dc}} \sim 0.01|J|-0.1|J|$ corresponds to $0.1-1$ $\mathrm{MV} / \mathrm{cm}$. We note that a similar dimerization $\left(J_{x} \neq J_{y}\right)$ can appear in a class of Kitaev candidates even without a dc electric field.

The Kitaev model (1) is exactly solvable via fermionization [58,61], as detailed in the literature [10-13,58,61] (see the supplemental material [57] for details). The fermionized Hamiltonian consists of four kinds of Majorana fermions: two dispersive and two localized ones (called visons). Visons have a small gap [58] and are absent [62] in the ground state at zero temperature $T=0$. The Hamiltonian at $T=0$ is therefore described only by the dispersive fermions. Figures $1(\mathrm{c})-1(\mathrm{~g})$ show that the dispersive fermion is gapless around the isotropic point $J_{x, y, z}=J$, while the $\kappa$ term opens a gap. We note that some perturbations can also be fermionized $[63,64]$, and therefore our analysis below is also applicable to such perturbed Kitaev models [65].

To consider HHG in the Kitaev model at $T=0$, we introduce an ac ME coupling between an ac electric field $E_{\text {ac }}(t)$ and the polarization $\hat{P}: H_{\mathrm{ms}}(t)=-E_{\mathrm{ac}}(t) \hat{P}$ [54]. We have assumed that $\boldsymbol{E}_{\text {ac }} \| \tilde{x}$, and the field is a Gaussian pulse with a THz frequency $\Omega: E_{\mathrm{ac}}(t)=E_{\mathrm{ac}} \cos (\Omega t) f(t)$, and the envelope function is given by $f(t)=\exp \left[-2(\ln 2)\left(t^{2} / t_{\mathrm{FWHM}}^{2}\right)\right]$, where $t_{\mathrm{FWHM}}$ is the full width at half-maximum of the intensity $E_{\mathrm{ac}}(t)^{2}$. We define the ac coupling constant $\mathcal{E}_{\mathrm{ac}}=E_{\mathrm{ac}} \eta_{\mathrm{ms}}$ and consider five-cycle pulses $\left(t_{\mathrm{FWHM}} / T=5\right)$. We note that even after application of $E_{\mathrm{ac}}(t)$, the Hamiltonian $H_{0}+H_{\mathrm{ms}}(t)$ is described by a bilinear form of the dispersive fermion in wave-vector $\boldsymbol{k}$ space. There is no vison dynamics in our setup that is valid in sufficiently low temperatures. We also phenomenologically introduce dissipation effects described by the Lindblad equation so as not to break the block-diagonal structure in $\boldsymbol{k}[41,46,55,56]$ (for details, see the supplemental material [57]). The dissipation is $\boldsymbol{k}$-independent and characterized by the dissipation rate $\gamma=0.1 J$, which corresponds to the relaxation time $\tau=1 / \gamma \sim 7.6 \mathrm{ps}$ for $J / k_{B}=10 \mathrm{~K}$ [66-75]. We suppose that the system is initially $(t \rightarrow-\infty)$ in the ground state, and we solve the Lindblad equation numerically to obtain the THz-driven nonequilibrium dynamics.

The observable of interest is $\hat{P}$, which is the source of HHG in our model. Time evolution of polarization is given by $P(t)=\langle\hat{P}\rangle_{t}=N^{-1} \sum_{k, k_{x}>0} \operatorname{Tr}\left[\rho(\boldsymbol{k}, t) P_{\boldsymbol{k}}\right]$, where $P_{\boldsymbol{k}}$ is $2 \times 2$ reduced polarization for the subspace with $\boldsymbol{k}$, and $N$ is the total number of unit cells. Since the electromagnetic radiation is proportional to $d^{2} P(t) / d t^{2}$, the radiation power at frequency $\omega$ is given by $I(\omega)=\left|\omega^{2} P(\omega)\right|^{2}$, where $P(\omega)$ is the Fourier 


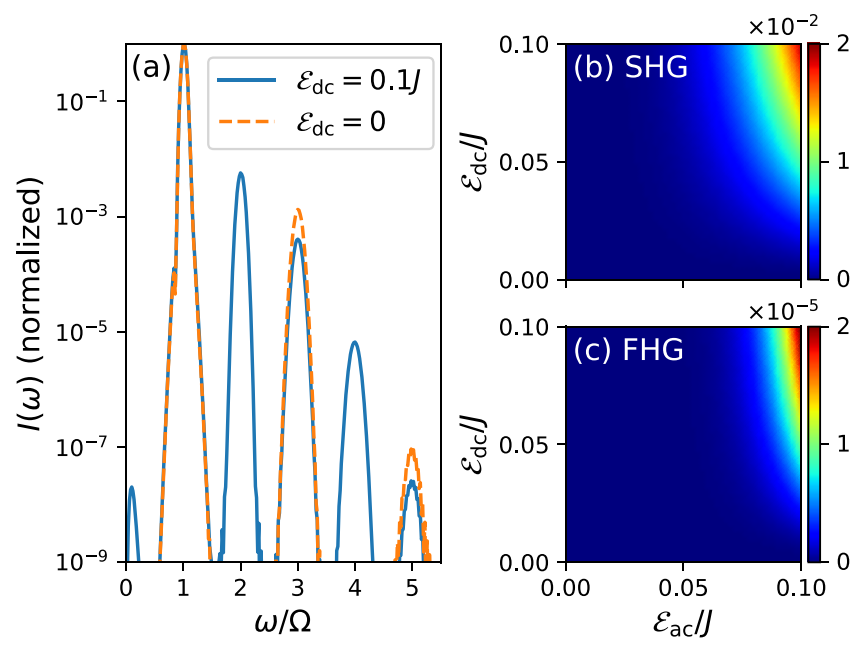

FIG. 2. HHG spectra $I(\omega)$ in driven isotropic $\left(J_{x, y, z}=J\right)$ Kitaev models with/without a dc electric field $E_{\mathrm{dc}}$ at $\kappa=0$ under a THz pulse of $\Omega=2.0 J$. (a) $I(\omega)$ as a function of $\omega$ at $\mathcal{E}_{\mathrm{dc}}=0$ and $0.1 J$ under the irradiation of $\mathcal{E}_{\mathrm{ac}}=0.1 J . I(\omega)$ is normalized with its maximum value. (b),(c) $\left(E_{\mathrm{ac}}, E_{\mathrm{dc}}\right)$ dependence of SHG $[I(2 \Omega)]$ and FHG $[I(4 \Omega)]$ spectra. The intensities, panels (b) and (c), are normalized with $I(\Omega)$ at $\mathcal{E}_{\mathrm{ac}}=\mathcal{E}_{\mathrm{dc}}=0.05 \mathrm{~J}$.

transform of $P(t)$ [76]. Since a constant shift of $P(t)$ does not change $I(\omega)$, we will also use $\Delta P(t)=P(t)-P\left(t_{\text {ini }}\right)$.

Effect of a dc Electric Field and Dimerization. We turn to our analyses and results. First we focus on the dc-electric-field dependence of HHG in the Kitaev model with $J_{x, y, z}=J$ and $\kappa=0$. Figure 2(a) shows a typical HHG spectrum $I(\omega)$ for ferromagnetic $(J>0)$ Kitaev models at $\mathcal{E}_{\mathrm{dc}}=0$ and $0.1 J$. Figures 2(b) and 2(c) are, respectively, the $\left(E_{\mathrm{ac}}, E_{\mathrm{dc}}\right)$ dependence of the SHG and fourth harmonic generation (FHG) that arise due to dimerization by $E_{\mathrm{dc}} \neq 0$. The SHG signal, activated by the dc electric field, is stronger than higher-order harmonics, and it can be a useful probe for QSL, as we discuss further below.

The HHG selection rules are often understood by dynamical symmetries that become exact in the limit of $t_{\text {FWHM }} \rightarrow \infty$ [77,78], also known as the time glide symmetry [79]. For our Kitaev model, we find that the following dynamical symmetry determines the HHG selection rule. In the nondimerized case of $E_{\mathrm{dc}}=0$, the Hamiltonian $H_{0}+H_{\mathrm{ms}}(t)$ is invariant under the time translation operation $t \rightarrow t+T / 2$ combined with the unitary transformation $\hat{U}=\hat{U}_{\text {mir }} \times \hat{U}_{\pi / 2}^{z}$, where $\hat{U}_{\text {mir }}$ is the reflection operation with respect to the $\tilde{y}$ axis and $\hat{U}_{\pi / 2}^{z}$ is the global $\pi / 2$ spin rotation around the $S^{z}$ axis. $\hat{P}$ is odd for this transformation, and therefore we obtain $P(t+T / 2)=-P(t)$, which means that even-order HHG is prohibited, consistent with Fig. 2 [57]. However, for $E_{\mathrm{dc}} \neq 0$, this dynamical symmetry is broken, and even-order HHG is allowed. We note that for $\kappa \neq 0$, the unitary operator $\hat{U}$ is modified as $\hat{U} \rightarrow \hat{V} \hat{U}$, where $\hat{V}$ is the time-reversal operator.

Thus, even-order HHG can be controlled by the static electric field $E_{\mathrm{dc}}$ through dimerization. Similar effects have been discussed in a spin chain [41] and in electronic systems [80-90], where $E_{\mathrm{dc}}$ induces electric current breaking the inversion symmetry. Being based only on symmetry, the dynamical
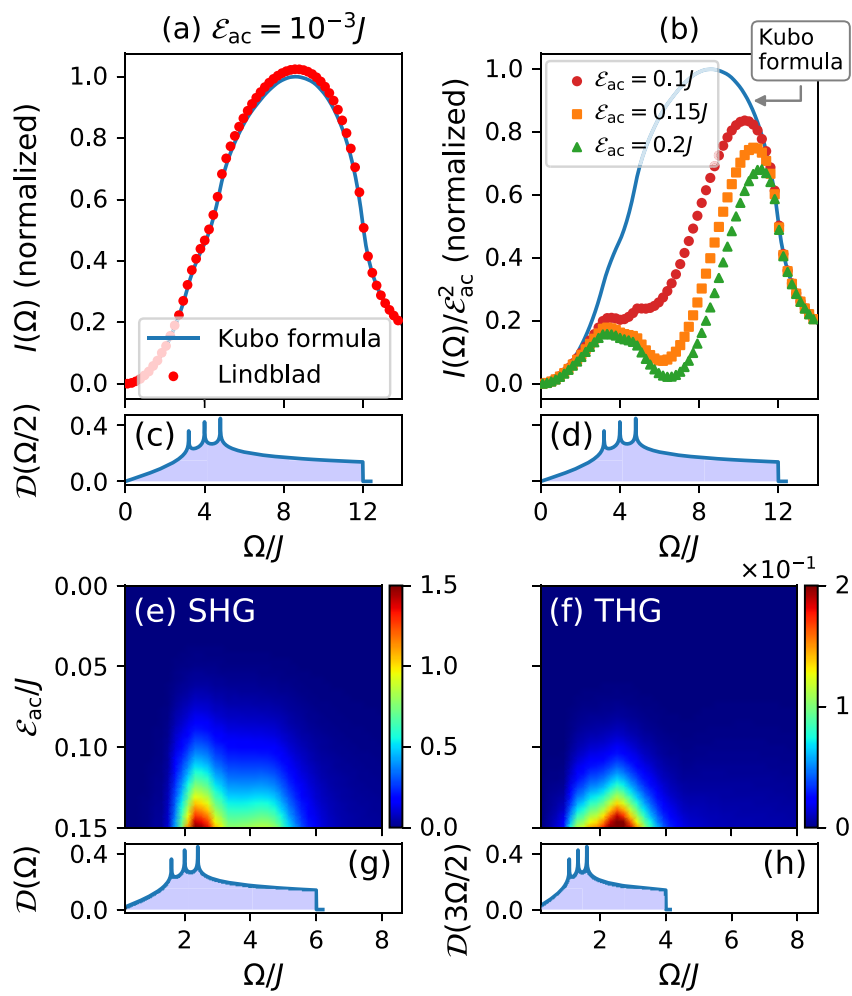

FIG. 3. $I(\Omega)$ of the Kitaev models with $J_{x, y, z}=J$ and $\mathcal{E}_{\text {dc }}=0.1 J$ at (a) a weak laser intensity $\mathcal{E}_{\mathrm{ac}} / J=10^{-3}$ and (b) strong intensities of $\mathcal{E}_{\text {ac }} / J=0.1,0.15$, and 0.2 . Blue lines and dotted points are, respectively, results of the linear response (Kubo) theory and numerically solved master equation [57]. $I(\Omega)$ is normalized with the maximum value of the Kubo formula. Panels (c) and (d) show DOSs of fermions corresponding to cases (a) and (b), respectively. (e) SHG $[I(2 \Omega)]$ and (f) THG $[I(3 \Omega)]$ of the Kitaev model with $\mathcal{E}_{\mathrm{dc}}=0.1 J$ in the space $\left(\mathcal{E}_{\text {ac }}, \Omega\right)$. The intensities, panels (e) and (f), are normalized with $I(\Omega)$ at $\left(\mathcal{E}_{\mathrm{ac}}, \Omega\right)=(0.05 J, 2 J)$. The corresponding DOSs are depicted in panels (g) and (h).

symmetry argument is applicable to a wide class of perturbed Kitaev models. For example, the dynamical symmetry survives for $J_{z} \neq J_{x}=J_{y}$ while it breaks down for $J_{x} \neq J_{y}$. The magnetic anisotropy dependence of even-order harmonics is discussed in the supplemental material [57]. In the following, we assume $E_{\mathrm{dc}} \neq 0$ as necessary to ensure that even-order HHG is present.

Dependence of Laser Frequency and Intensity. Next, we consider the $\Omega$ and $E_{\text {ac }}$ dependence of the HHG. Figures 3(a) and 3(b), respectively, show the $\Omega$ dependence of $I(\Omega)$ for weak $\left(\mathcal{E}_{\mathrm{ac}}=10^{-3} J\right)$ and strong $\left(\mathcal{E}_{\mathrm{ac}}=0.1 J, 0.15 J, 0.2 J\right) \mathrm{THz}$ pulses. In addition to the numerical result of the Lindblad equation, we plot that of the linear response theory (the Kubo formula) (see the supplemental material [57]). From the comparison between $I(\Omega)$ in Fig. 3(a) [Fig. 3(b)] and the fermion density of state (DOS) $\mathcal{D}(\omega)$ in Fig. 3(c) [Fig. 3(d)], we find that two-particle continuum spectra occur in the driven Kitaev model. This results from the ME coupling between an ac electric field (i.e., photon) and a pair of fermions with $\boldsymbol{k}$ and $\boldsymbol{- k}$. This continuum indicates the existence of fermionic excitations in Kitaev magnets, and it differs qualitatively from usual 
ordered magnets, where one often observes a $\delta$-functional peak due to magnons [91].

It is noteworthy that even the fundamental harmonic $(\omega=$ $\Omega$ ) shows characteristics of the QSL in the strong THz pulse. Unlike in the weak pulse, the Kubo formula is no longer valid in the strong one in the deep nonperturbative regime. In this regime of $\mathcal{E}_{\text {ac }} \gtrsim 0.15 \mathrm{~J}$, a new broad peak emerges in $I(\Omega)$ at $\Omega_{\text {peak }} \sim 4 J$ [Fig. 3(b)], which is twice as large as the high DOS position. Namely, $I(\Omega)$ driven by intense pulses tells us the peak position of the DOS. For instance, $E_{\mathrm{ac}}=$ $0.15 J / \eta_{\mathrm{ms}} \sim 3 \mathrm{MV} / \mathrm{cm}$ for $J=10 \mathrm{~K}$ under the assumption of $\mathcal{E}_{\mathrm{ac}}=g_{0} \mu_{B} E_{\mathrm{ac}} / c$, and it indicates that the currently available $\mathrm{THz}$ laser is strong enough to observe such nonlinear optical spectra. We note that in Fig. 3(b), the increase of $I(\Omega)$ in the high- $\Omega$ range around $\Omega \sim 8 J$ is due to the factor $\Omega^{4}$ in $I(\Omega)$.

The SHG and third harmonic generation (THG) spectra, $I(2 \Omega)$ and $I(3 \Omega)$, are depicted in Figs. 3(e) and 3(f). We find that broad peaks in $I(2 \Omega)$ and $I(3 \Omega)$ appear around $\Omega=$ $\Omega_{\text {peak }} / 2$ and $\Omega_{\text {peak }} / 3$, respectively. This is a natural result, indicating that excitation processes creating fermions with a high DOS are dominant in HHG. Figure 3(h) shows that the peak frequency of the THG is slightly higher than that of the $\operatorname{DOS} \mathcal{D}(3 \Omega / 2)$. This would also be attributed to the factor $(3 \Omega)^{4}$ in $I(3 \Omega)$.

Effect of a dc Magnetic Field. Now we discuss the dcmagnetic-field dependence of HHG in Kitaev models. We focus on the antiferromagnetic Kitaev model [57], where the Kitaev QSL state is more stable against magnetic fields than in the ferromagnetic case [92]. The magnetic-field driven $\kappa$ term opens a mass gap $\Delta_{\kappa}$ in the fermion band, as shown in Fig. 1(g). As $\Delta_{\kappa}$ increases, the maximum of $\mathcal{D}(\omega)$ at $\omega \sim 2|J|$ grows up for $\Delta_{\kappa} \lesssim 2|J|(\kappa \lesssim 0.2|J|)$ [see Figs. 1(e) and $1(\mathrm{~g})$ ]. Therefore, the intensities of HHG spectra are expected to be controlled by the dc magnetic field and laser frequency $\Omega$. Figure 4 proves this expectation. Panels (a) and (c), respectively, show the $\Omega$ dependence of $I(\Omega)$ and $\mathcal{D}(\omega)$ at a tuned effective magnetic field $\kappa=0.2|J|$, where $\mathcal{D}(\Omega)$ take the highest value at $\Omega_{0} \sim \Delta_{\kappa} \sim 2|J|$. In this case, we have a sharp peak of $I(\Omega)$ at $\Omega_{\text {peak }}=2 \Omega_{0} \sim 4|J|$. Figures 4(b) and 4(d) represent $I(\Omega)$ and $\mathcal{D}(\omega)$ as functions of $\kappa$ at a fixed $\Omega=2|J|$. We see that a clear peak of $I(\Omega)$ appears when $\Delta_{\kappa}$ passes across one-half of the laser frequency $\Omega / 2$.

We also show $I(\Omega)$ and $I(2 \Omega)$ in the $(\kappa, \Omega)$ space in Figs. 4(e) and 4(f). For $I(\Omega)$ in the case of $\kappa \gtrsim 0.2|J|$, the frequency $\Omega$ of the broad peak increases monotonically in an almost $\kappa$-linear fashion. This is because the peak position $\Omega_{0}$ of $\mathcal{D}(\Omega)$ increases almost linearly with $\kappa$ for $\kappa \gtrsim 0.2|J|$ (for more details, see Fig. S15 of the supplemental material [57]). Since $\kappa \sim|\boldsymbol{B}|^{3}$, the $\boldsymbol{B}$-cube-dependent frequency at the peak is specific for the Kitaev model and essentially differs from the $\boldsymbol{B}$-linear behavior of magnon peaks. The frequency at the peak of $I(2 \Omega)$ is almost half of $\Omega_{\text {peak }}$ of $I(\Omega)$, as shown in Fig. 4(f), and this is a natural result from the perturbative viewpoint. We note that, even in the linear response regime ( $\omega=\Omega$ and weak $\mathrm{THz}$ pulse), the fundamental harmonic shows characteristics of the QSL for $\kappa \gtrsim 0.2|J|$ (see the supplemental material [57]). $I(\Omega)$ driven by intense pulses with a finite magnetic field tells us the peak position of the DOS $\Omega_{0}$, which is half as large as $\Omega_{\text {peak }}$.
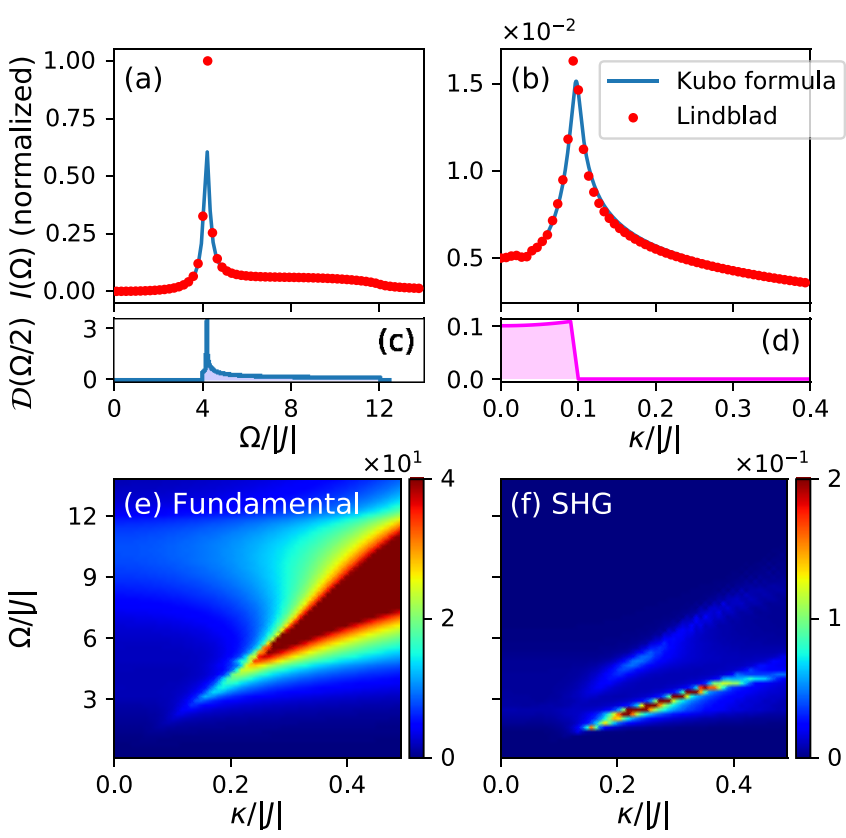

FIG. 4. (a) $I(\Omega)$ in an antiferromagnetic $(J<0)$ Kitaev model with $\kappa=0.2|J|, \mathcal{E}_{\mathrm{dc}}=0$, and $\mathcal{E}_{\mathrm{ac}}=10^{-3}|J|$. (b) $\kappa$ dependence of $I(\Omega)$ in $\Omega=2.0|J|, \mathcal{E}_{\mathrm{dc}}=0$, and $\mathcal{E}_{\mathrm{ac}}=10^{-3}|J|$. Blue line and dotted points are, respectively, results of the linear response theory and the master equation [57]. $I(\Omega)$ in (a) and (b) are normalized with the maximum value in panel (a). (c) $\Omega$ and (d) $\kappa$ dependences of $\mathcal{D}(\omega)$ that, respectively, correspond to panels (a) and (b). (e) $I(\Omega)$ and (f) $I(2 \Omega)$ of the Kitaev model with $\mathcal{E}_{\mathrm{dc}}=\mathcal{E}_{\mathrm{ac}}=0.1|J|$. The intensities, panels (e) and (f), are normalized with $I(\Omega)$ at $(\kappa, \Omega)=$ $(0.05|J|, 2|J|)$.

Finally, we estimate the laser intensity required in the HHG experiment. For the Kitaev magnet with $|J| / k_{B}=10 \mathrm{~K}$ and $\kappa=0$, the required ac electric field $E_{\mathrm{ac}}$ for the observation of HHG can be estimated from Fig. $2(\mathrm{a}): E_{\mathrm{ac}}=2.6 \mathrm{MV} / \mathrm{cm}$ at $0.42 \mathrm{THz}$ is necessary for $R(\Omega)=I(2 \Omega) / I(\Omega) \gtrsim 10^{-2}$ and $E_{\mathrm{ac}}=0.9 \mathrm{MV} / \mathrm{cm}$ for $R(\Omega) \gtrsim 10^{-3}$. These required ac electric fields can be reduced when applying the effective static magnetic field $\kappa=0.2|J|$ as shown in Fig. 4 and the supplemental material [57]. In this case, the electric field is estimated as $E_{\mathrm{ac}}=0.7 \mathrm{MV} / \mathrm{cm}$ at $0.42 \mathrm{THz}$ for $R(\Omega) \gtrsim 10^{-2}$ and $E_{\mathrm{ac}}=0.2 \mathrm{MV} / \mathrm{cm}$ for $R(\Omega) \gtrsim 10^{-3}$. We remark that the above criteria, $R(\Omega) \gtrsim 10^{-2}$ and $10^{-3}$, for successful detection are much more strict than those in the actual experiments for electronic systems [51-53]. Thus, much weaker pulses might be enough to verify our predictions experimentally. These estimates indicate that lower-order harmonics in Kitaev magnets can be detected with current $\mathrm{THz}$ light techniques.

Conclusions. We have analyzed the HHG in Kitaev magnets with an ME coupling by using a quantum master equation and the linear response theory. Our results show that the specific nature of the Majorana fermion excitations can be detected by linear and nonlinear THz-light responses. The characteristics of the Kitaev model, such as even-order harmonics, continuum HHG spectra, and broad peaks, can be controlled by applying static electric or magnetic fields. This 
study sheds light on an interdisciplinary field between photoscience and QSLs.

Our setup does not accompany vison (localized fermion) excitations. Studies for laser-driven vison dynamics and the effects of temperature and ac Zeeman terms are interesting directions for future work.
Acknowledgments. M.S. was supported by JSPS KAKENHI (Grants No. 17K05513 and No. 20H01830) and a Grant-in-Aid for Scientific Research on Innovative Areas "Quantum Liquid Crystals" (Grant No. JP19H05825). T.N.I. was supported by JSPS KAKENHI Grant No. JP18K13495 and No. 21K13852.
[1] X.-G. Wen, Quantum Field Theory of Many-Body Systems (Oxford University Press, Oxford, 2007).

[2] L. Balents, Spin liquids in frustrated magnets, Nature 464, 199 (2010).

[3] L. Savary and L. Balents, Quantum spin liquids: a review, Rep. Prog. Phys. 80, 016502 (2017).

[4] J. Knolle and R. Moessner, A field guide to spin liquids, Annu. Rev. Condens. Matter Phys. 10, 451 (2019).

[5] M. Yamashita, N. Nakata, Y. Senshu, M. Nagata, H. M. Yamamoto, R. Kato, T. Shibauchi, and Y. Matsuda, Highly mobile gapless excitations in a two-dimensional candidate quantum spin liquid, Science 328, 1246 (2010).

[6] A. V. Sologubenko, K. Giannò, H. R. Ott, A. Vietkine, and A. Revcolevschi, Heat transport by lattice and spin excitations in the spin-chain compounds $\mathrm{SrCuO}_{2}$ and $\mathrm{Sr}_{2} \mathrm{CuO}_{3}$, Phys. Rev. B 64, 054412 (2001).

[7] D. Hirobe, M. Sato, Y. Shiomi, H. Tanaka, and E. Saitoh, Magnetic thermal conductivity far above the Néel temperature in the Kitaev-magnet candidate $\alpha-\mathrm{RuCl}_{3}$, Phys. Rev. B 95, 241112(R) (2017).

[8] Y. Onose, T. Ideue, H. Katsura, Y. Shiomi, N. Nagaosa, and Y. Tokura, Observation of the magnon hall effect, Science 329, 297 (2010).

[9] D. Watanabe, K. Sugii, M. Shimozawa, Y. Suzuki, T. Yajima, H. Ishikawa, Z. Hiroi, T. Shibauchi, Y. Matsuda, and M. Yamashita, Emergence of nontrivial magnetic excitations in a spin-liquid state of kagomé volborthite, Proc. Natl. Acad. Sci. (USA) 113, 8653 (2016).

[10] S. Trebst, Kitaev materials, arXiv:1701.07056.

[11] M. Hermanns, I. Kimchi, and J. Knolle, Physics of the kitaev model: fractionalization, dynamic correlations, and material connections, Annu. Rev. Condens. Matter Phys. 9, 17 (2018).

[12] H. Takagi, T. Takayama, G. Jackeli, G. Khaliullin, and S. E. Nagler, Concept and realization of Kitaev quantum spin liquids, Nat. Rev. Phys. 1, 264 (2019).

[13] Y. Motome and J. Nasu, Hunting majorana fermions in kitaev magnets, J. Phys. Soc. Jpn. 89, 012002 (2020).

[14] Y. Motome, R. Sano, S. Jang, Y. Sugita, and Y. Kato, Materials design of Kitaev spin liquids beyond the Jackeli-Khaliullin mechanism, J. Phys. Condens. Matter 32, 404001 (2020).

[15] Y. Kasahara, T. Ohnishi, Y. Mizukami, O. Tanaka, S. Ma, K. Sugii, N. Kurita, H. Tanaka, J. Nasu, Y. Motome, T. Shibauchi, and Y. Matsuda, Majorana quantization and half-integer thermal quantum Hall effect in a Kitaev spin liquid, Nature 559, 227 (2018).

[16] L. J. Sandilands, Y. Tian, K. W. Plumb, Y.-J. Kim, and K. S. Burch, Scattering Continuum and Possible Fractionalized Excitations in $\alpha-\mathrm{RuCl}_{3}$, Phys. Rev. Lett. 114, 147201 (2015).

[17] J. Nasu, J. Knolle, D. L. Kovrizhin, Y. Motome, and R. Moessner, Fermionic response from fractionalization in an insulating two-dimensional magnet, Nat. Phys. 12, 912 (2016).
[18] J. Xing, E. Feng, Y. Liu, E. Emmanouilidou, C. Hu, J. Liu, D. Graf, A. P. Ramirez, G. Chen, H. Cao, and N. Ni, Néel-type antiferromagnetic order and magnetic field-temperature phase diagram in the spin- $\frac{1}{2}$ rare-earth honeycomb compound $\mathrm{YbCl}_{3}$, Phys. Rev. B 102, 014427 (2020).

[19] H. Hirori, A. Doi, F. Blanchard, and K. Tanaka, Single-cycle terahertz pulses with amplitudes exceeding $1 \mathrm{MV} / \mathrm{cm}$ generated by optical rectification in $\mathrm{LiNbO}_{3}$, Appl. Phys. Lett. 98, 091106 (2011).

[20] M. Sato, T. Higuchi, N. Kanda, K. Konishi, K. Yoshioka, T. Suzuki, K. Misawa, and M. Kuwata-Gonokami, Terahertz polarization pulse shaping with arbitrary field control, Nat. Photon. 7, 724 (2013).

[21] S. S. Dhillon, M. S. Vitiello, E. H. Linfield, A. G. Davies, M. C. Hoffmann, J. Booske, C. Paoloni, M. Gensch, P. Weightman, G. P. Williams, E. Castro-Camus, D. R. S. Cumming, F. Simoens, I. Escorcia-Carranza, J. Grant, S. Lucyszyn, M. Kuwata-Gonokami, K. Konishi, M. Koch, C. A. Schmuttenmaer, T. L. Cocker, R. Huber, A. G. Markelz, Z. D. Taylor, V. P. Wallace, J. Axel Zeitler, J. Sibik, T. M. Korter, B. Ellison, S. Rea, P. Goldsmith, K. B. Cooper, R. Appleby, D. Pardo, P. G. Huggard, V. Krozer, H. Shams, M. Fice, C. Renaud, A. Seeds, A. Stöhr, M. Naftaly, N. Ridler, R. Clarke, J. E. Cunningham, and M. B. Johnston, The 2017 terahertz science and technology roadmap, J. Phys. D 50, 043001 (2017).

[22] B. Liu, H. Bromberger, A. Cartella, T. Gebert, M. Först, and A. Cavalleri, Generation of narrowband, high-intensity, carrierenvelope phase-stable pulses tunable between 4 and $18 \mathrm{THz}$, Opt. Lett. 42, 129 (2017).

[23] A. Pimenov, A. A. Mukhin, V. Y. Ivanov, V. D. Travkin, A. M. Balbashov, and A. Loidl, Possible evidence for electromagnons in multiferroic manganites, Nat. Phys. 2, 97 (2006).

[24] Y. Takahashi, R. Shimano, Y. Kaneko, H. Murakawa, and Y. Tokura, Magnetoelectric resonance with electromagnons in a perovskite helimagnet, Nat. Phys. 8, 121 (2012).

[25] T. Kubacka, J. A. Johnson, M. C. Hoffmann, C. Vicario, S. de Jong, P. Beaud, S. Grubel, S. W. Huang, L. Huber, L. Patthey, Y. D. Chuang, J. J. Turner, G. L. Dakovski, W. S. Lee, M. P. Minitti, W. Schlotter, R. G. Moore, C. P. Hauri, S. M. Koohpayeh, V. Scagnoli, G. Ingold, S. L. Johnson, and U. Staub, Large-amplitude spin dynamics driven by a THz pulse in resonance with an electromagnon, Science 343, 1333 (2014).

[26] I. Kézsmárki, D. Szaller, S. Bordács, V. Kocsis, Y. Tokunaga, Y. Taguchi, H. Murakawa, Y. Tokura, H. Engelkamp, T. Rõõm, and U. Nagel, One-way transparency of four-coloured spin-wave excitations in multiferroic materials, Nat. Commun. 5, 3203 (2014).

[27] Y. Mukai, H. Hirori, T. Yamamoto, H. Kageyama, and K. Tanaka, Nonlinear magnetization dynamics of antiferromagnetic spin resonance induced by intense terahertz magnetic field, New J. Phys. 18, 013045 (2016). 
[28] S. Baierl, J. H. Mentink, M. Hohenleutner, L. Braun, T.-M. Do, C. Lange, A. Sell, M. Fiebig, G. Woltersdorf, T. Kampfrath, and R. Huber, Terahertz-Driven Nonlinear Spin Response of Antiferromagnetic Nickel Oxide, Phys. Rev. Lett. 117, 197201 (2016).

[29] J. Lu, X. Li, H. Y. Hwang, B. K. Ofori-Okai, T. Kurihara, T. Suemoto, and K. A. Nelson, Coherent Two-Dimensional Terahertz Magnetic Resonance Spectroscopy of Collective Spin Waves, Phys. Rev. Lett. 118, 207204 (2017).

[30] P. Němec, M. Fiebig, T. Kampfrath, and A. V. Kimel, Antiferromagnetic opto-spintronics, Nat. Phys. 14, 229 (2018).

[31] A. A. Sirenko, P. Marsik, C. Bernhard, T. N. Stanislavchuk, V. Kiryukhin, and S.-W. Cheong, Terahertz Vortex Beam as a Spectroscopic Probe of Magnetic Excitations, Phys. Rev. Lett. 122, 237401 (2019).

[32] S. Miyahara and N. Furukawa, Theory of electric field induced one-magnon resonance in cycloidal spin magnets, arXiv:0811.4082.

[33] M. Mochizuki and N. Nagaosa, Theoretically Predicted Picosecond Optical Switching of Spin Chirality in Multiferroics, Phys. Rev. Lett. 105, 147202 (2010).

[34] M. Mochizuki, N. Furukawa, and N. Nagaosa, Theory of Electromagnons in the Multiferroic Mn Perovskites: The Vital Role of Higher Harmonic Components of the Spiral Spin Order, Phys. Rev. Lett. 104, 177206 (2010).

[35] S. Miyahara and N. Furukawa, Nonreciprocal directional dichroism and toroidal magnons in helical magnets, J. Phys. Soc. Jpn. 81, 023712 (2012).

[36] S. Takayoshi, H. Aoki, and T. Oka, Magnetization and phase transition induced by circularly polarized laser in quantum magnets, Phys. Rev. B 90, 085150 (2014).

[37] S. Takayoshi, M. Sato, and T. Oka, Laser-induced magnetization curve, Phys. Rev. B 90, 214413 (2014).

[38] M. Sato, Y. Sasaki, and T. Oka, Floquet Majorana edge mode and non-Abelian anyons in a driven kitaev model, arXiv:1404.2010.

[39] M. Sato, S. Takayoshi, and T. Oka, Laser-Driven Multiferroics And Ultrafast Spin Current Generation, Phys. Rev. Lett. 117, 147202 (2016).

[40] H. Fujita and M. Sato, Encoding orbital angular momentum of light in magnets, Phys. Rev. B 96, 060407(R) (2017).

[41] T. N. Ikeda and M. Sato, High-harmonic generation by electric polarization, spin current, and magnetization, Phys. Rev. B 100, 214424 (2019).

[42] H. Ishizuka and M. Sato, Rectification Of Spin Current In Inversion-Asymmetric Magnets With Linearly Polarized Electromagnetic Waves, Phys. Rev. Lett. 122, 197702 (2019).

[43] H. Ishizuka and M. Sato, Theory for shift current of bosons: Photogalvanic spin current in ferrimagnetic and antiferromagnetic insulators, Phys. Rev. B 100, 224411 (2019).

[44] S. Higashikawa, H. Fujita, and M. Sato, Floquet engineering of classical systems, arXiv:1810.01103.

[45] S. Takayoshi, Y. Murakami, and P. Werner, High-harmonic generation in quantum spin systems, Phys. Rev. B 99, 184303 (2019).

[46] M. Sato and Y. Morisaku, Two-photon driven magnon-pair resonance as a signature of spin-nematic order, Phys. Rev. B 102, 060401(R) (2020).
[47] Y. Wan and N. P. Armitage, Resolving Continua Of Fractional Excitations By Spinon Echo In THz 2D Coherent Spectroscopy, Phys. Rev. Lett. 122, 257401 (2019).

[48] W. Choi, K. H. Lee, and Y. B. Kim, Theory Of TwoDimensional Nonlinear Spectroscopy For The Kitaev Spin Liquid, Phys. Rev. Lett. 124, 117205 (2020).

[49] S. Ghimire, A. D. Dichiara, E. Sistrunk, P. Agostini, L. F. Dimauro, and D. A. Reis, Observation of high-order harmonic generation in a bulk crystal, Nat. Phys. 7, 1847 (2011).

[50] O. Schubert, M. Hohenleutner, F. Langer, B. Urbanek, C. Lange, U. Huttner, D. Golde, T. Meier, M. Kira, S. W. Koch, and R. Huber, Sub-cycle control of terahertz high-harmonic generation by dynamical Bloch oscillations, Nat. Photon. 8, 119 (2014).

[51] H. A. Hafez, S. Kovalev, J.-C. Deinert, Z. Mics, B. Green, N. Awari, M. Chen, S. Germanskiy, U. Lehnert, J. Teichert, Z. Wang, K.-J. Tielrooij, Z. Liu, Z. Chen, A. Narita, K. Müllen, M. Bonn, M. Gensch, and D. Turchinovich, Extremely efficient terahertz high-harmonic generation in graphene by hot Dirac fermions, Nature 561, 507 (2018).

[52] B. Cheng, N. Kanda, T. N. Ikeda, T. Matsuda, P. Xia, T. Schumann, S. Stemmer, J. Itatani, N. P. Armitage, and R. Matsunaga, Efficient Terahertz Harmonic Generation With Coherent Acceleration Of Electrons In The Dirac Semimetal $\mathrm{Cd}_{3} \mathrm{As}_{2}$, Phys. Rev. Lett. 124, 117402 (2020).

[53] S. Kovalev, R. M. A. Dantas, S. Germanskiy, J.-C. Deinert, B. Green, I. Ilyakov, N. Awari, M. Chen, M. Bawatna, J. Ling, F. Xiu, P. H. M. van Loosdrecht, P. Surówka, T. Oka, and Z. Wang, Non-perturbative terahertz high-harmonic generation in the three-dimensional Dirac semimetal $\mathrm{Cd}_{3} \mathrm{As}_{2}$, Nat. Commun. 11, 2451 (2020).

[54] Y. Tokura, S. Seki, and N. Nagaosa, Multiferroics of spin origin, Rep. Prog. Phys. 77, 076501 (2014).

[55] H.-P. Breuer and F. Petruccione, The Theory of Open Quantum Systems (Oxford University Press, Oxford, 2007).

[56] R. Alicki and K. Lendi, Quantum Dynamical Semigroups and Applications, Lecture Notes in Physics Vol. 717 (Springer, Berlin, 2007).

[57] See Supplemental Material at http://link.aps.org/supplemental/ 10.1103/PhysRevResearch.3.L032024 for additional details, such as the magnetic anisotropy dependence of even-order harmonics and the results in the ferromagnetic $(J>0)$ Kitaev model.

[58] A. Kitaev, Anyons in an exactly solved model and beyond, Ann. Phys. 321, 2 (2006).

[59] D. Hüvonen, U. Nagel, T. Rõõm, Y. J. Choi, C. L. Zhang, S. Park, and S.-W. Cheong, Magnetic excitations and optical transitions in the multiferroic spin- $\frac{1}{2}$ system $\mathrm{LiCu}_{2} \mathrm{O}_{2}$, Phys. Rev. B 80, 100402(R) (2009).

[60] S. Furukawa, M. Sato, and S. Onoda, Chiral Order And Electromagnetic Dynamics In One-Dimensional Multiferroic Cuprates, Phys. Rev. Lett. 105, 257205 (2010).

[61] H.-D. Chen and Z. Nussinov, Exact results of the Kitaev model on a hexagonal lattice: spin states, string and brane correlators, and anyonic excitations, J. Phys. A 41, 075001 (2008).

[62] E. H. Lieb, Flux Phase Of The Half-Filled Band, Phys. Rev. Lett. 73, 2158 (1994). 
[63] D. Takikawa and S. Fujimoto, Impact of off-diagonal exchange interactions on the Kitaev spin-liquid state of $\alpha-\mathrm{RuCl}_{3}$, Phys. Rev. B 99, 224409 (2019).

[64] D. Takikawa and S. Fujimoto, Topological phase transition to Abelian anyon phases due to off-diagonal exchange interaction in the Kitaev spin liquid state, Phys. Rev. B 102, 174414 (2020).

[65] As discussed in Ref. [63], if the Kitaev magnet has both a dc magnetic field and a sort of perturbative term, an additional three spin interaction appears and its coupling constant is possibly proportional to $B$. However, this paper has focused on the system with $\kappa \propto B^{3}$.

[66] A. Kirilyuk, A. V. Kimel, and T. Rasing, Ultrafast optical manipulation of magnetic order, Rev. Mod. Phys. 82, 2731 (2010).

[67] M. Oshikawa and I. Affleck, Low-Temperature Electron Spin Resonance Theory For Half-Integer Spin Antiferromagnetic Chains, Phys. Rev. Lett. 82, 5136 (1999).

[68] M. Oshikawa and I. Affleck, Electron spin resonance in $S=\frac{1}{2}$ antiferromagnetic chains, Phys. Rev. B 65, 134410 (2002).

[69] S. C. Furuya and M. Sato, Electron spin resonance in quasione-dimensional quantum antiferromagnets: relevance of weak interchain interactions, J. Phys. Soc. Jpn. 84, 033704 (2015).

[70] E. Beaurepaire, J.-C. Merle, A. Daunois, and J.-Y. Bigot, Ultrafast Spin Dynamics In Ferromagnetic Nickel, Phys. Rev. Lett. 76, 4250 (1996).

[71] B. Koopmans, M. van Kampen, J. T. Kohlhepp, and W. J. M. de Jonge, Ultrafast Magneto-Optics In Nickel: Magnetism Or Optics?, Phys. Rev. Lett. 85, 844 (2000).

[72] K. Lenz, H. Wende, W. Kuch, K. Baberschke, K. Nagy, and A. Jánossy, Two-magnon scattering and viscous Gilbert damping in ultrathin ferromagnets, Phys. Rev. B 73, 144424 (2006).

[73] C. Vittoria, S. D. Yoon, and A. Widom, Relaxation mechanism for ordered magnetic materials, Phys. Rev. B 81, 014412 (2010).

[74] E. A. Mashkovich, K. A. Grishunin, R. V. Mikhaylovskiy, A. K. Zvezdin, R. V. Pisarev, M. B. Strugatsky, P. C. M. Christianen, T. Rasing, and A. V. Kimel, Terahertz Optomagnetism: Nonlinear THz Excitation Of GHz Spin Waves In Antiferromagnetic $\mathrm{FeBO}_{3}$, Phys. Rev. Lett. 123, 157202 (2019).

[75] C. Tzschaschel, T. Satoh, and M. Fiebig, Tracking the ultrafast motion of an antiferromagnetic order parameter, Nat. Commun. 10, 3995 (2019).

[76] J. D. Jackson, Classical Electrodynamics (Wiley, Weinheim, Germany, 1998).

[77] O. E. Alon, V. Averbukh, and N. Moiseyev, Selection Rules For The High Harmonic Generation Spectra, Phys. Rev. Lett. 80, 3743 (1998).

[78] O. Neufeld, D. Podolsky, and O. Cohen, Floquet group theory and its application to selection rules in harmonic generation, Nat. Commun. 10, 405 (2019).
[79] T. Morimoto, H. C. Po, and A. Vishwanath, Floquet topological phases protected by time glide symmetry, Phys. Rev. B 95, 195155 (2017).

[80] J. B. Khurgin, Current induced second harmonic generation in semiconductors, Appl. Phys. Lett. 67, 1113 (1995).

[81] S. Wu, L. Mao, A. M. Jones, W. Yao, C. Zhang, and X. Xu, Quantum-enhanced tunable second-order optical nonlinearity in bilayer graphene, Nano Lett. 12, 2032 (2012).

[82] J. L. Cheng, N. Vermeulen, and J. E. Sipe, DC current induced second order optical nonlinearity in graphene, Opt. Express 22 , 15868 (2014)

[83] O. A. Aktsipetrov, V. O. Bessonov, A. A. Fedyanin, and V. O. Val'dner, DC-induced generation of the reflected second harmonic in silicon, JETP Lett. 89, 58 (2009).

[84] B. A. Ruzicka, L. K. Werake, G. Xu, J. B. Khurgin, E. Y Sherman, J. Z. Wu, and H. Zhao, Second-Harmonic Generation Induced By Electric Currents In GaAs, Phys. Rev. Lett. 108 077403 (2012)

[85] A. Y. Bykov, T. V. Murzina, M. G. Rybin, and E. D. Obraztsova, Second harmonic generation in multilayer graphene induced by direct electric current, Phys. Rev. B 85, 121413(R) (2012).

[86] Y. Q. An, F. Nelson, J. U. Lee, and A. C. Diebold, Enhanced optical second-harmonic generation from the current-biased graphene/ $\mathrm{SiO}_{2} / \mathrm{Si}(001)$ structure, Nano Lett. 13, 2104 (2013).

[87] A. Moor, A. F. Volkov, and K. B. Efetov, Amplitude Higgs Mode And Admittance In Superconductors With A Moving Condensate, Phys. Rev. Lett. 118, 047001 (2017).

[88] S. Nakamura, Y. Iida, Y. Murotani, R. Matsunaga, H. Terai, and R. Shimano, Infrared Activation Of The Higgs Mode By Supercurrent Injection In Superconducting NbN, Phys. Rev. Lett. 122, 257001 (2019).

[89] S. Nakamura, K. Katsumi, H. Terai, and R. Shimano, Nonreciprocal Terahertz Second-Harmonic Generation In Superconducting $\mathrm{NbN}$ under Supercurrent Injection, Phys. Rev. Lett. 125, 097004 (2020).

[90] K. Takasan, T. Morimoto, J. Orenstein, and J. E. Moore, Current-induced second harmonic generation in inversionsymmetric Dirac and Weyl semimetals, arXiv:2007.08887.

[91] Even a single-magnon spectrum can have a broad peak when its lifetime is short. In such a case, it becomes difficult to distinguish the Kitaev continuum and the broad magnon peak. However, combining the HHG experiment with an additional one, we can possibly distinguish these two excitations. For example, magnons always appear in an ordered phase which can be detected by a magnetic Bragg peak, a magnetic phase transition, etc.

[92] J. Nasu, Y. Kato, Y. Kamiya, and Y. Motome, Successive Majorana topological transitions driven by a magnetic field in the Kitaev model, Phys. Rev. B 98, 060416(R) (2018). 